\title{
Identifying a Generic and Detrimental Role of Fano Resonance in Spin Generation in Semiconductor Nanostructures
}

\author{
Y. Q. Huang, ${ }^{*}$ J. Beyer®, Y. Puttisong, I. A. Buyanova $\odot$, and W. M. Chen $\odot^{\dagger}$ \\ Department of Physics, Chemistry and Biology, Linköping University, S-58183 Linköping, Sweden
}

(Received 3 May 2021; revised 18 July 2021; accepted 19 August 2021; published 13 September 2021)

\begin{abstract}
Fano resonance is a fundamental physical process that strongly affects the electronic transport, optical, and vibronic properties of matter. Here, we provide the first experimental demonstration of its profound effect on spin properties in semiconductor nanostructures. We show that electron spin generation in InAs/ GaAs quantum-dot structures is completely quenched upon spin injection from adjacent InGaAs wetting layers at the Fano resonance due to coupling of light-hole excitons and the heavy-hole continuum of the interband optical transitions, mediated by an anisotropic exchange interaction. Using a master equation approach, we show that such quenching of spin generation is robust and independent of Fano parameters. This work therefore identifies spin-dependent Fano resonance as a universal spin loss channel in quantumdot systems with an inherent symmetry-breaking effect.
\end{abstract}

DOI: 10.1103/PhysRevLett.127.127401

Fano resonance (FR) emerges as a result of quantum interference between two spectrally overlapping pathways - one channel through a discrete level and the other via continuum states [1]. The interference leads to a characteristic asymmetric spectral line shape derived from destructive and constructive interference of the two pathways with rapid changing of phase shift in the vicinity of the FR. FR has been found in a wide variety of physical systems with coexisting discrete and continuum states, including electronic transport in low-dimensional materials [2,3], optical response of photonic devices and metamaterials [4-6], and interactions in phononic systems [7-9].

In semiconductor nanostructures, FR is frequently encountered when bound excitation, e.g., an exciton, overlaps with a dispersed energy band, which greatly reshapes the optical and transport properties of the nanostructures [10-12]. The interference of excitonic or electronic states, accompanied by their spin wave function, is expected to lead to intriguing physics and device innovation for future spintronics and opto-spintronics. For instance, there has been a theoretical proposal that FR can realize functionality of a perfect spin filter [13-15] in which transmission of unwanted spin orientation is suppressed upon destructive interference of a spin-dependent FR. Despite their anticipated importance, the effects of FR on spin properties remain largely unexplored. Limited early studies of spin-dependent FR are restricted to theoretical

Published by the American Physical Society under the terms of the Creative Commons Attribution 4.0 International license. Further distribution of this work must maintain attribution to the author(s) and the published article's title, journal citation, and DOI. Funded by Bibsam. calculations and have mostly focused on spin-dependent transport [13,15-17]. Up to now, however, a direct experimental determination of the FR effect on spin properties of semiconductor nanostructures and of semiconductors in general is still elusive.

Here, we provide the first experimental evidence for an important and direct role of FR in optical spin generation in semiconductor nanostructures. Using InAs/GaAs quantumdot (QD) structures (QDS) as a sensitive spin detector, we show that optical generation of electron spin polarization in a nearby ultrathin quantum well is completely quenched when circularly polarized excitation is tuned in resonance with FR formed between a light-hole exciton (XL) and a continuum of the band-to-band (BB) transition between the electron and heavy-hole (HH) subband (e-HH). The observed quenching of optical spin generation is accompanied by the presence of a giant anisotropic exchange interaction (AEI) of the XL, which suggests an effect of localization and symmetry breaking due to the dot-well interaction. Applying a master equation, we show that the effect arises from destructive interference of electron spin states induced by FR, which occurs independent of the Fano parameters. Our work thus identifies that spindestructive FR can be a robustly realized spin-loss channel in semiconductor nanostructures with a seemingly inherent strong anisotropy.

Table I lists the set of self-assembled InAs/GaAs QDS of different geometries that are investigated in this work. It includes single QD (SQD), two samples of double QD (DQD1 and DQD2), and quantum-dot cluster (QC). The AFM images and photoluminescence (PL) of the QDS can be found in Figs. S1 and S2 in the Supplemental Material [18]. All the QDS were grown by molecular beam epitaxy 
TABLE I. Summary of the WL exciton energies, HH-LH splitting $\Delta_{\mathrm{HH}-\mathrm{LH}}$ and XL fine-structure splitting $\Delta_{\mathrm{FSS}}^{\mathrm{XL}}$ in the studied QDS, measured at $5 \mathrm{~K}$. The emission energies of QDS $E_{\mathrm{QDS}}$ used in PLE measurements are also given.

\begin{tabular}{cccccrc}
\hline \hline QDS & $\begin{array}{c}E_{\mathrm{XH}} \\
\mathrm{eV}\end{array}$ & $\begin{array}{c}E_{\mathrm{XL}(y)} \\
\mathrm{eV}\end{array}$ & $\begin{array}{c}E_{\mathrm{XL}(x)} \\
\mathrm{eV}\end{array}$ & $\begin{array}{c}\Delta_{\mathrm{HH}-\mathrm{LH}} \\
\mathrm{meV}\end{array}$ & $\begin{array}{c}\left|\Delta_{\mathrm{FSS}}^{\mathrm{XL}}\right| \\
\mathrm{meV}\end{array}$ & $\begin{array}{c}E_{\mathrm{QDS}} \\
\mathrm{eV}\end{array}$ \\
\hline SQD & 1.445 & 1.485 & 1.481 & 39.6 & 3.6 & 1.081 \\
QC & 1.423 & 1.464 & 1.470 & 46.7 & 6.4 & 1.170 \\
DQD1 & 1.383 & 1.449 & 1.439 & 66.4 & 10.4 & 1.241 \\
DQD2 & 1.376 & 1.445 & 1.437 & 68.9 & 7.8 & 1.190 \\
\hline \hline
\end{tabular}

under the Stranski-Krastanov mode, with a 1.8-monolayer thin, coherently strained InAs quantum well, i.e., a wetting layer (WL), being formed right beneath the QDS layer. A detailed description of the growth parameters can be found elsewhere [19]. The energy alignment of the QDS is schematically illustrated in Fig. 1(a) and calculated in Fig. S3 in the Supplemental Material. The dot-well structure facilitates a sensitive detection of electron spins generated in the WL by monitoring spin polarization of the electrons injected to the nearby QDS.

As illustrated in Fig. 1(b), optical absorption in WL consists of (1) the continuum of the BB transitions between
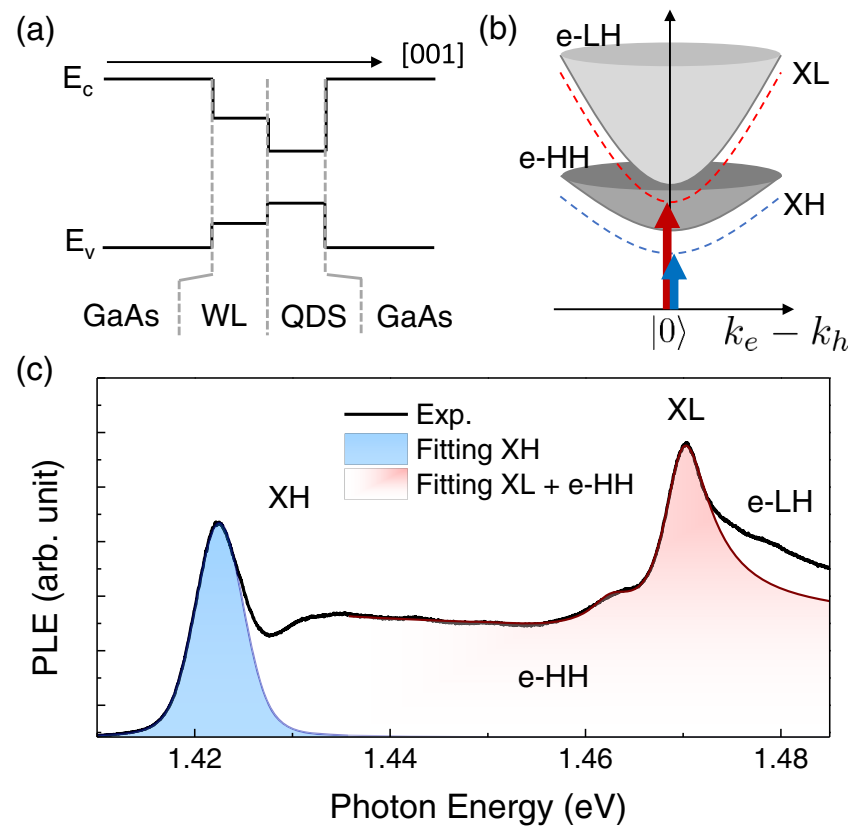

FIG. 1. (a) Schematic diagram of the structure and energy alignment of the QDS grown along the [0 00 l 1 ]axis. (b) Illustration of the optical excitation to XH and XL at different energies. Resonant excitation of XL overlaps with excitation of the e-HH continuum. (c) PLE spectrum detecting QC emission measured at $5 \mathrm{~K}$ by scanning laser excitation photon energy over the interband spectral range of the WL. The XH resonance is fitted with a Voigt line shape, while the sum of the XL and e-HH contributions is fitted with a Fano line shape given in Eq. (2). the electron subband and the HH or light-hole (LH) subband, denoted as e-HH or e- $\mathrm{LH}$, respectively, and (2) the discrete transitions from the corresponding $\mathrm{HH}$ and $\mathrm{LH}$ excitons, denoted as XL and XH. Figure 1(c) shows a representative PL excitation (PLE) spectrum measured over the spectral range of the WL absorption by detecting the PL intensity of the $\mathrm{QC}$ ensemble at $5 \mathrm{~K}$ arising from carrier or exciton transfer from the WL. It reveals the aforementioned contributions as illustrated in Fig. 1(b). XH at the lowest energy is excluded from any overlap with the e- $\mathrm{HH}$ and e- $\mathrm{LH}$ continuum absorption such that FR is absent. It can be fitted well by a Voigt line shape (see the Supplemental Material) with the corresponding Gaussian and Lorentzian broadening of $w_{G}=5.2 \mathrm{meV}$ and $w_{L}=1.1 \mathrm{meV}$, respectively, likely as a result of inhomogeneous and homogeneous broadening. In contrast, XL spectrally overlaps with the e-HH continuum and exhibits an asymmetric line shape, which hints the occurrence of FR.

Electron spin polarization can be generated in WL with the so-called optical orientation technique [20], as illustrated in Fig. 2(a). Circularly polarized excitation, e.g., $\sigma^{+}$, creates spin-up $\left(m_{s}=+\frac{1}{2}\right)$ and spin-down $\left(m_{s}=-\frac{1}{2}\right)$ conduction band $(\mathrm{CB})$ electrons under the interband excitation involving $\mathrm{LH}$ and $\mathrm{HH}$, respectively. Thanks to the different oscillator strengths between the $\mathrm{LH}$ and $\mathrm{HH}$ BB transitions, the photogenerated electrons acquire a net spin polarization and can subsequently be injected to a nearby QDS. Meanwhile, the hole spin is generally believed to be completely depolarized due to a strong spin-orbit coupling [21]. The measurement geometry of the optical orientation is schematically shown in the right panel of Fig. 2(b) and a more detailed description of the measurements is given in the method section of the Supplemental Material. We monitor such spin injection process by measuring the $\sigma^{ \pm}$-polarized PL components (denoted as $I^{\sigma^{ \pm}}$) at the emission energy of the QDS ( $E_{\mathrm{QDS}}$ in Table I) while scanning excitation photon energy across the XL resonances. The resulting circular polarization $P_{\mathrm{PL}}^{\text {cir. }}=I^{\sigma^{+}}-$ $I^{\sigma^{-}} / I^{\sigma^{+}}+I^{\sigma^{-}}$is displayed in Figs. 2(d)-2(f). When the excitation photon energy is below XL where only e- $\mathrm{HH}$ is involved, $P_{\mathrm{PL}}^{\text {cir. }}=-2\left\langle s_{z}\right\rangle$ directly measures the injected electron spin projection $\left\langle s_{z}\right\rangle$ in the QDS. At and above $\mathrm{XL}$ when both e-HH and e-LH participate and generate electrons of opposite spin orientation, $P_{\mathrm{PL}}^{\text {cir. }}$ is expected to be reduced as a result of compensation between these two competing spin generation channels. Surprisingly, in all QDS samples studied in this work regardless of their difference in shape and emission energies, electron spin polarization is negligible at low temperature when the excitation is resonant with $\mathrm{XL}$. This is evident from the vanishing $P_{\mathrm{PL}}^{\text {cir. }}$ under both $\sigma^{+}$and $\sigma^{-}$excitation as marked by the solid arrows in Figs. 2(d)-2(f). The observed vanishing $\left\langle s_{z}\right\rangle$ in all QDS samples can only be explained by complete quenching of optical spin generation in WL, 


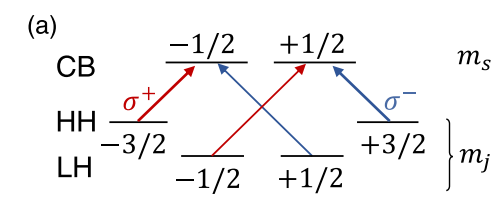

(b)

(d)
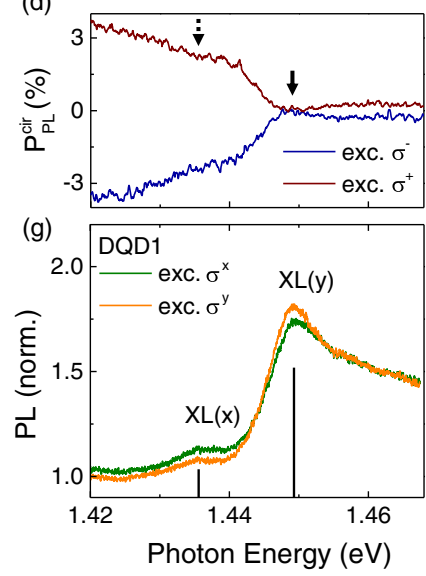

(e)
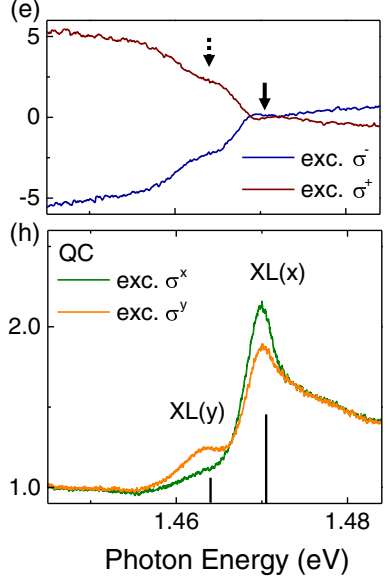

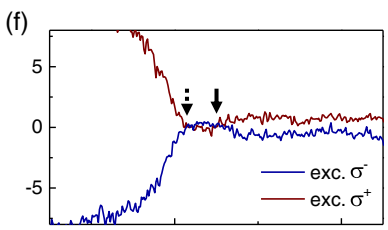

(i) SQD

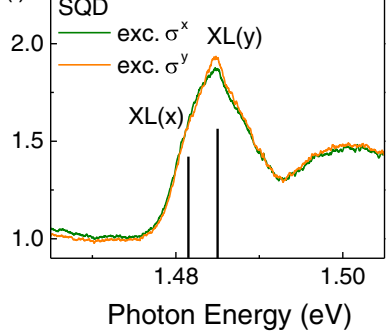

FIG. 2. (a) Selection rules of the BB transitions. (b) Measurement geometry under linearly (left panel) and circularly (right panel) polarized excitation. (c) PL of QC by excitation at the two XL PLE peaks shown in (h) as a function of rotation angle of the excitation

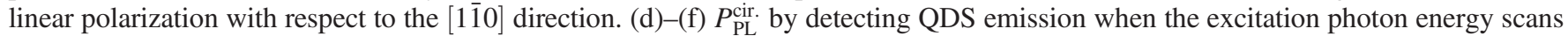
across the XL resonances of DQD1, QC, and SQD under circularly polarized excitation. (g)-(i) PLE spectra under linearly polarized excitation with the excitation polarization along either the [ $\left.\begin{array}{lll}1 & 1 & 0\end{array}\right](x)$ or $\left[\begin{array}{ll}1 & 1\end{array}\right]$ ( $\left.y\right)$ axis, by detecting unpolarized PL from the QDS. All the experimental data were obtained at $5 \mathrm{~K}$.

rather than accidental compensation of electron spin polarization between the optical excitation involving $\mathrm{HH}$ and LH. Such compensation effect critically depends on the relative PLE intensity between e-HH and XL, which varies among the studied QDS [see Figs. 2(g)-2(i)] such that the condition for complete cancellation should not be fulfilled for all. A detailed estimation of the compensation effect is given in the Supplemental Material.

To identify the physical mechanism for the complete quenching of spin generation at XL FR, we closely examine the spin properties of XL by employing PLE spectroscopy with linearly polarized excitation $\left(\sigma^{x}\right.$ and $\left.\sigma^{y}\right)$ following the measurement geometry shown in the left panel of Fig. 2(b). Here, the $x$ and $y$ axes are defined along the $\left[\begin{array}{lll}1 & 1 & 0\end{array}\right]$ and $\left[\begin{array}{ll}1 & 10\end{array}\right]$ crystallographic axes of the substrate. Figures 2(g)-2(i) show the PLE spectra measured by monitoring unpolarized PL emissions from the DQD1, QC, and SQD samples under $\sigma^{x}$ and $\sigma^{y}$ excitation. XL is found to consist of two bright exciton states, which are split in energy (marked by the vertical lines) and have different orientations of dipole moment (denoted as $\mathrm{XL}(x)$ and $\mathrm{XL}(y)$ by their linear polarization direction). In Fig. 2(c), $\mathrm{PL}$ intensity of QC under the resonant excitation of $\mathrm{XL}(x)$ and $\mathrm{XL}(y)$ of WL (after removing a background contribution from excitation of e- $\mathrm{HH}$ ) is plotted as a function of the excitation linear polarization direction. It confirms that the dipole moments of the two XL resonances are orthogonal and primarily along the two $\langle 110\rangle$ axes, which resembles the fine-structure splitting (FSS) commonly seen for excitons in QDs due to AEI [22]. However, the FSS for an ideal $2 \mathrm{D}$ exciton should vanish at $\Gamma$ point [23]. The observed FSS of XL is therefore attributed to the presence of a local in-plane anisotropy such that XL is subjected to
AEI. We note that local confinement and symmetry breaking have previously been established for $\mathrm{XH}$ in $\mathrm{WL}$ in the vicinity of a QDS [24]. In view of the commonly accepted fact that QDs suffer in-plane anisotropy in shape, strain, chemical compositions, etc., it is not surprising that local areas of WL in direct contact with QDs also experience corresponding in-plane anisotropy. The observed strong AEI is expected to lead to a spin-mixed XL such that electron spin generation via XL is not plausible. However, as e-HH does not suffer AEI, a parallel spin generation channel via e-HH spectrally overlapping with XL is still capable of generating of finite spin polarization if there is no coupling between XL and e-HH. The general observation of complete quenching of spin generation at the $\mathrm{XL}$ resonance must then stem from FR between e-HH and XL.

An effective Hamiltonian describing both effects of AEI and Fano interaction can be written with the basis of optically allowed XL states $\left|\psi_{\mathrm{XL}}^{\sigma}\right\rangle$ and e-HH continuum state $\left|\varphi_{E_{k}}^{\sigma}\right\rangle$ as

$$
\begin{aligned}
H_{0}= & \sum_{\sigma, \sigma^{\prime}} H_{\sigma, \sigma^{\prime}}^{\mathrm{XL}}\left|\psi_{\mathrm{XL}}^{\sigma}\right\rangle\left\langle\psi_{\mathrm{XL}}^{\sigma^{\prime}}\left|+\sum_{\sigma, \boldsymbol{k}} E_{\boldsymbol{k}}\right| \varphi_{E_{k}}^{\sigma}\right\rangle\left\langle\varphi_{E_{k}}^{\sigma}\right| \\
& +\sum_{\sigma, \boldsymbol{k}} V_{H}(\boldsymbol{k})\left|\varphi_{E_{k}}^{\sigma}\right\rangle\left\langle\psi_{\mathrm{XL}}^{\sigma}\right|+\text { H.c. }
\end{aligned}
$$

where $\sigma, \sigma^{\prime}=\uparrow$ or $\downarrow$ denotes electron spin only. $E_{k}$ is the kinetic energy of the e-HH continuum state. $V_{H}(\boldsymbol{k})$ is the $\boldsymbol{k}$ dependent HH-XL coupling, which stems from coupling of the hole subband. We replace $V_{H}(\boldsymbol{k})$ with $V_{H}\left(E_{\boldsymbol{k}}\right)$, which disregards for now the $\boldsymbol{k}$-space orientation $\left(\theta_{\boldsymbol{k}}\right)$, and discuss its effect at the end. $H_{\sigma, \sigma^{\prime}}^{\mathrm{XL}}=E_{\mathrm{XL}} \delta_{\sigma, \sigma^{\prime}}+J \tau_{x}$ with $E_{\mathrm{XL}}$ and $J$ being the exciton energy and strength of the AEI. $\tau_{x}$ is the Pauli matrix operating on the spin subspace of XL. 
We consider the optical spin generation under the framework of a density matrix formalism. The details of the derivation are given in the Supplemental Material. The PLE spectra under $\sigma^{-}$excitation are calculated from the steady-state density matrix $\rho$, which can be cast to a familiar form of FR:

$$
\operatorname{Tr}\{\rho\} \propto \sum_{\eta=x, y} \int d E \frac{\left|q_{\eta}+\epsilon_{\eta}\right|^{2}}{1+\epsilon_{\eta}^{2}} \frac{\Gamma / \Gamma_{1}\left|D_{\mathrm{HH}}\right|^{2}}{(\hbar \omega-E)^{2}+\hbar^{2} \Gamma^{2}} .
$$

Here, $\epsilon_{\eta}=\left(E-E_{\eta}-F\right) / \pi\left|V_{H}(E)\right|^{2}$ is the dimensionless energy with respect to the $\mathrm{XL}(x)$ or $\mathrm{XL}(y)$ resonance energy $\left(E_{x}\right.$ or $\left.E_{y}\right) . F$ is the coupling induced FR shift same as in Ref. [1]. $D_{\mathrm{HH}}, D_{\mathrm{XL}}^{x}$, and $D_{\mathrm{XL}}^{y}$ are respectively the dipole moment element of e-HH, $\mathrm{XL}(x)$, and $\mathrm{XL}(y)$ under circularly polarized excitation. $\Gamma$ is the broadening parameter associated with optical excitation. $\Gamma_{1}$ represents relaxation of WL transitions, which includes contributions from recombination and injection to QDS. The Fano parameter $q_{\eta}=\left[D_{\mathrm{XL}}^{\eta} / D_{\mathrm{HH}}+\int d E_{\boldsymbol{k}} V_{H}\left(E_{k}\right) / E-E_{\boldsymbol{k}}\right] / \pi V_{H}(E) \quad$ is sensitive to the oscillator strengths of $\mathrm{XL}$ and e-HH transitions and also HH-LH coupling. Equation (2) confirms our experimental observation that the PLE spectra show two FR features that occur at the two XL states split by AEI.

The optical spin generation is obtained with $\left\langle s_{z}\right\rangle=$ $\operatorname{Tr}\left\{\rho s_{z}\right\} / \operatorname{Tr}\{\rho\}$. Considering dephasing of optical excitation only for energy degenerated states of $\Gamma_{2}$, one obtains

$$
\operatorname{Tr}\left\{\rho s_{z}\right\} \propto \int d E\left|D_{\mathrm{HH}}\right|^{2}\left[\frac{\epsilon_{x} \epsilon_{y}+1}{\left(1+\epsilon_{x}^{2}\right)\left(1+\epsilon_{y}^{2}\right)} \operatorname{Re}\left\{\left(q_{x}+\epsilon_{x}\right)\left(q_{y}^{*}+\epsilon_{y}\right)\right\}\right] \frac{2 \Gamma / \Gamma_{2}}{(\hbar \omega-E)^{2}+\hbar^{2} \Gamma^{2}} .
$$

For a large enough AEI (e.g., measured by the effective AEI $\left.\quad \epsilon_{x e}=J / \pi\left|V_{H}\left(E_{k}\right)\right|^{2}>1\right), \quad \epsilon_{x} \epsilon_{y}+1=0 \quad$ would always have two real solutions at $\epsilon= \pm \sqrt{\epsilon_{x e}^{2}-1}$, where $\epsilon=\left(\epsilon_{x}+\epsilon_{y}\right) / 2$. In the absence of a broadening effect, it leads to vanishing optical spin generation that is independent of the Fano parameter.

The robust quenching of spin generation can be understood as a result of destructive interference of spin wave function at the FR. Because of the spin degeneracy, excitation of the e-HH continuum states with circularly polarized light creates two spin-mixed states $\left|\varphi_{E_{k}}^{x}\right\rangle$ and $\left|\varphi_{E_{k}}^{y}\right\rangle$ coherently. Spin generation via the e-HH continuum critically depends on their relative phase difference. As illustrated in Fig. 3(a), the FR at two XL resonances independently introduces a phase shift $\Delta_{x}$ and $\Delta_{y}$ for $\left|\varphi_{E_{k}}^{x}\right\rangle$ and $\left|\varphi_{E_{k}}^{y}\right\rangle . \Delta_{x}\left(\Delta_{y}\right)$ develops a rapid $\pi$-phase change at around $\mathrm{XL}(x)[\mathrm{XL}(y)]$ resonance, which guarantees a destructive interference of the spin wave function at $\Delta_{x}-\Delta_{y}= \pm \pi / 2$.

In the above discussion, $V_{H}(\boldsymbol{k})$ is treated independently of $\theta_{k}$. The HH-LH coupling may alter for different $\theta_{k}$. For instance, in Luttinger-Kohn Hamiltonian [25], coupling of $\mathrm{HH}$ and LH states with the same spin takes the form $V_{H}(\boldsymbol{k}) \propto\left(\mu_{1} e^{2 i \theta_{k}}-\mu_{2} e^{-2 i \theta_{k}}\right) \boldsymbol{k}^{2}$, where $\mu_{1} \gg \mu_{2}$ is found for InAs. This is approximated by a phase factor $e^{2 i \theta_{k}}$ added to $V_{H}(\boldsymbol{k})$ and $q_{\eta}$. In Fig. 3(b), we show the simulated $\left\langle s_{z}\right\rangle$ as a function of $\epsilon$ for $\theta_{k}=0 \rightarrow \pi$, which shows that $\theta_{k}$ has a strong effect on the optical spin generation as evident from the variation of $\left\langle s_{z}\right\rangle$ in the vicinity of the FR. Still, the predicted quenching of spin generation as marked by the dashed line remains intact. In Figs. 3(c) and 3(d), $\langle\rho\rangle$ and $\left\langle s_{z}\right\rangle$ are calculated after integration over $\theta_{\boldsymbol{k}}$, which shows robust quenching of $\left\langle s_{z}\right\rangle$ as identified by our experiment.
We note that the quenching of spin generation can be shadowed by a broadening effect included in Eq. (3). This is shown by the simulation results in Fig. 4(a) when an effective broadening parameter $\gamma=\hbar \Gamma / \pi\left|V_{H}(\boldsymbol{k})\right|^{2}$ is varied from 0.1 to 0.4 . The effect becomes more severe for the lower-energy XL resonance in DQD1 and QC (see Fig. 2), which is attributed to a weaker contribution of the excitonic transition and a larger linewidth (see Fig. S7 in the Supplemental Material). Furthermore, we have also observed a recovery of optical spin generation at $T>100 \mathrm{~K}$. The temperature trend of $P_{\mathrm{PL}}^{\text {cir. }}$ is correlated with an increase of homogenous broadening $w_{L}$ of the (a)

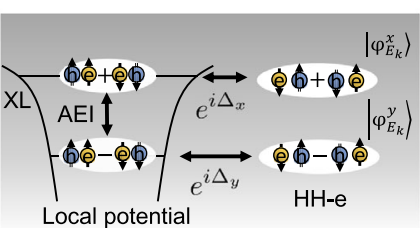

(b)

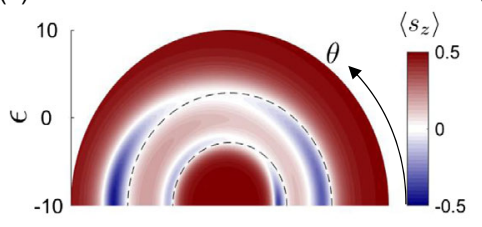

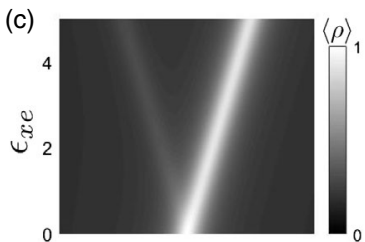

(d)

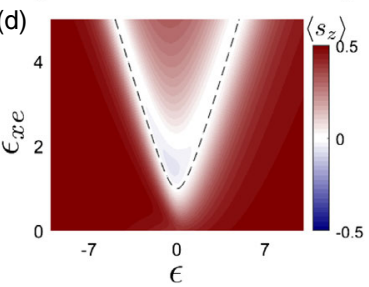

FIG. 3. (a) Illustration of the two $\mathrm{FR}$ at $\mathrm{XL}(x)$ and $\mathrm{XL}(y)$ resonance and the phase shift $\Delta_{x}$ and $\Delta_{y}$ associated with the FR. (b) Simulated $\left\langle s_{z}\right\rangle$ in the absence of Lorentzian broadening for $q_{x}=1.5 e^{i \theta}, q_{y}=3 e^{i \theta}, \epsilon_{x e}=3$. (c) and (d) are the simulated normalized $\langle\rho\rangle$ and $\left\langle s_{z}\right\rangle$ in the absence of Lorentzian broadening for $q_{x}=1.5 e^{2 i \theta}, q_{y}=3 e^{2 i \theta}, \epsilon_{x e}=3$ after integration of $\theta$ from 0 to $2 \pi$. In (b) and (d), the dashed line marks the predicted robust quenching of spin generation at $\epsilon= \pm \sqrt{\epsilon_{x e}^{2}-1}$. 

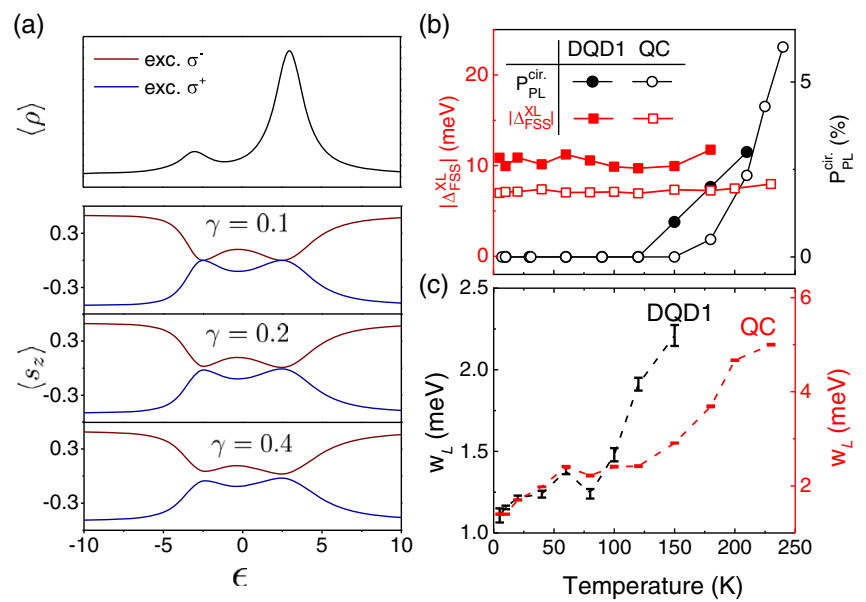

FIG. 4. (a) Simulation of $\left\langle s_{z}\right\rangle$ (lower panel) for different values of $\gamma$. Upper panel shows $\langle\rho\rangle$ at $\gamma=0.1$. (b) Temperature dependent trend of the $\left|\Delta_{\mathrm{FSS}}^{\mathrm{XL}}\right|$ (the red left axis) and $P_{\mathrm{PL}}^{\mathrm{cir}}$. (the black right axis) under resonant excitation of the XL FR for the QC (the open symbols) and DQD1 (the filled symbols) samples. (c) Temperature dependent trend of the Lorentzian broadening $w_{L}$ estimated from the Voigt line shape fitting of the $\mathrm{XH}$ resonance from the QC and DQD1 samples.

exciton resonances as deduced from the Voigt line shape fitting of XH from QC and DQD1 in Figs. 4(b) and 4(c). We anticipate that $w_{L}$ of $\mathrm{XH}$ gives a reasonable estimation of the XL broadening since the inhomogeneous linewidth $w_{G}$ of XL is expected to be small. This is shown in Fig. S4 in the Supplemental Material by a comparison of the PLE spectra detecting from different individual QCs. At the same time, the magnitude of the XL FSS, $\left|\Delta_{\mathrm{FSS}}^{\mathrm{XL}}\right|$, is not noticeably changed over the entire temperature range.

To conclude, we have provided the first direct experimental proof for a profound impact of FR on spin properties, in particular electron spin generation, in a variety of InAs/GaAs nanostructures. FR coupling between the $\mathrm{XL}$ and e-HH continuum leads to complete quenching of electron spin generation regardless of the large differences between the studied QDS. This quenching is shown to originate from the combined effect of FR and strong AEI of XL, responsible for a coherently destructive interference of the electron spin wave function. Both experimental and theoretical investigations have demonstrated that the FR-induced complete quenching of spin generation is robust as it is independent of the FR parameter. Our work has thus identified the important role of FR in spin properties of semiconductor nanostructures, e.g., a universal spin-loss channel, that could be tailored for spintronic applications.

We thank S. Suraprapapich and C. W. Tu for providing the QDS samples and Mikhail M. Glazov, Mikhail Durnev, E. L. Ivchenko, and B. Sernelius for valuable discussion. This work is supported by the Swedish Research Council
(Grants No. 2016-05091 and 2020-04530) and the Swedish Government Strategic Research Area in Materials Science on Functional Materials at Linköping University (Faculty Grant SFO-Mat-LiU \# 2009-00971).

* Corresponding author. yuqing.huang@liu.se Corresponding author. weimin.chen@liu.se

[1] U. Fano, Phys. Rev. 124, 1866 (1961).

[2] J. Göres, D. Goldhaber-Gordon, S. Heemeyer, M. A. Kastner, H. Shtrikman, D. Mahalu, and U. Meirav, Phys. Rev. B 62, 2188 (2000).

[3] F. Borsoi, K. Zuo, S. Gazibegovic, R. L. M. Op het Veld, E. P. A. M. Bakkers, L. P. Kouwenhoven, and S. Heedt, Nat. Commun. 11, 3666 (2020).

[4] A. Bärnthaler, S. Rotter, F. Libisch, J. Burgdörfer, S. Gehler, U. Kuhl, and H.-J. Stöckmann, Phys. Rev. Lett. 105, 056801 (2010).

[5] Y. Wang, L. Liao, T. Hu, S. Luo, L. Wu, J. Wang, Z. Zhang, W. Xie, L. Sun, A. V. Kavokin, X. Shen, and Z. Chen, Phys. Rev. Lett. 118, 063602 (2017).

[6] F. Zangeneh-Nejad and R. Fleury, Phys. Rev. Lett. 122, 014301 (2019).

[7] T.-T. Tang, Y. Zhang, C.-H. Park, B. Geng, C. Girit, Z. Hao, M. C. Martin, A. Zettl, M. F. Crommie, S. G. Louie, Y. R. Shen, and F. Wang, Nat. Nanotechnol. 5, 32 (2010).

[8] M. L. Kerfoot, A. O. Govorov, C. Czarnocki, D. Lu, Y. N. Gad, A. S. Bracker, D. Gammon, and M. Scheibner, Nat. Commun. 5, 3299 (2014).

[9] B. Xu, Y. M. Dai, L. X. Zhao, K. Wang, R. Yang, W. Zhang, J. Y. Liu, H. Xiao, G. F. Chen, S. A. Trugman, J.-X. Zhu, A. J. Taylor, D. A. Yarotski, R. P. Prasankumar, and X. G. Qiu, Nat. Commun. 8, 14933 (2017).

[10] C. P. Holfeld, F. Löser, M. Sudzius, K. Leo, D. M. Whittaker, and K. Köhler, Phys. Rev. Lett. 81, 874 (1998).

[11] S. Bar-Ad, P. Kner, M. V. Marquezini, S. Mukamel, and D. S. Chemla, Phys. Rev. Lett. 78, 1363 (1997).

[12] A. E. Miroshnichenko, S. Flach, and Y.S. Kivshar, Rev. Mod. Phys. 82, 2257 (2010).

[13] J. F. Song, Y. Ochiai, and J. P. Bird, Appl. Phys. Lett. 82, 4561 (2003).

[14] M. E. Torio, K. Hallberg, S. Flach, A. E. Miroshnichenko, and M. Titov, Eur. Phys. J. B 37, 399 (2003).

[15] C.-R. Liu, L. Huang, H. Luo, and Y.-C. Lai, Phys. Rev. Applied 13, 034061 (2020).

[16] F. Zhai and H. Q. Xu, Phys. Rev. B 76, 035306 (2007).

[17] K. P. Wójcik and I. Weymann, Phys. Rev. B 90, 115308 (2014).

[18] See Supplemental Material at http://link.aps.org/ supplemental/10.1103/PhysRevLett.127.127401 for descriptions on method, effect of spin relaxation, estimation of compensation effect, broadening of XL resonances, Voigt line shape, derivation of equations, AFM and PL spectra and calculated electronic structure of WL and QDS.

[19] S. Suraprapapich, S. Panyakeow, and C. W. Tu, Appl. Phys. Lett. 90, 183112 (2007). 
[20] F. Meier and B. Zakharchenya, Optical Orientation (North Holland, Amsterdam, 1984).

[21] M. I. Dyakonov, Spin Physics in Semiconductors (Springer Berlin Heidelberg, Berlin, Heidelberg, 2008).

[22] Y. H. Huo, B. J. Witek, S. Kumar, J. R. Cardenas, J. X. Zhang, N. Akopian, R. Singh, E. Zallo, R. Grifone, D. Kriegner, R. Trotta, F. Ding, J. Stangl, V. Zwiller,
G. Bester, A. Rastelli, and O. G. Schmidt, Nat. Phys. 10, 46 (2014).

[23] L. C. Andreani and F. Bassani, Phys. Rev. B 41, 7536 (1990).

[24] Y. Huang, Y. Puttisong, I. A. Buyanova, and W. M. Chen, Nano Res. 9, 602 (2016).

[25] D. A. Broido and L. J. Sham, Phys. Rev. B 31, 888 (1985). 